\title{
Antigenic Diversity of the Hepatitis B Virus Capsid
} Audray Harris* $\neq 1$, David Belnap ${ }^{2}$, Norman Watts ${ }^{1}$, James Conway ${ }^{3}$, Naiqian Cheng ${ }^{1}$, Stephen Stahl ${ }^{1}$, Paul Wingfield ${ }^{1}$ and Alasdair Steven ${ }^{1}$

Address: ${ }^{1}$ NIAMS-NIH, Bethesda, MD 20892, ${ }^{2}$ Brigham Young University, Provo, UT 84602 and ${ }^{3}$ University of Pittsburgh, PA 15261

* Corresponding author $\ddagger$ Presenting author

from 2005 International Meeting of The Institute of Human Virology

Baltimore, USA, 29 August - 2 September 2005

Published: 8 December 2005

Retrovirology 2005, 2(Suppl I):P35 doi:10.1 I86/1742-4690-2-SI-P35

Antibodies to the capsid (cAg, core antigen) of HBV play a central role in the immune response to this virus. Since most anti-cAg antibodies recognize conformational epitopes, ruling out characterization by immunochemical methods, we have approached the problem by cryo-EM and image reconstruction of Fab-decorated capsids, combined with molecular modelling. Recently, we characterized the epitopes for two anti-cAg monoclonals ( $\mathrm{mAb} 88$, $\mathrm{mAb} 842$ ). Both Fabs engage sites on the protruding capsid spikes, but they differ in binding orientation and are distinct. Occupancies of symmetry-related potential binding sites are unequal, reflecting prefererential binding to certain quasiequivalent variants. Despite steric interference, it was possible to identify both epitopes. The epitope for IgG mAb 88, residues 77-87, consists of an alpha-helix on one of the polypeptide chains within the dimeric spike. That of IgM mAb 842, also conformational, consists of loops and helical regions on both polypeptide chains, residues $74-78$ and $78-83$ and duplicates a previously characterized epitope. To date, six epitopes have been characterized, one being linear and five conformational. Although accessible sites are limited to two small regions of the capsid surface, combinations of a small set of motifs generate substantial antigenic diversity. We estimate the total number of distinct epitopes to be about 20 . 